\title{
Moderate hypothermia induces protein SUMOylation in bone marrow stromal cells and enhances their tolerance to hypoxia
}

\author{
WENBO REN ${ }^{1,2}$, XIAOFANG MA ${ }^{3}$, XIAOZHI LIU ${ }^{3}$, YANXIA LI $^{3}$, \\ ZHONGMIN JIANG ${ }^{4}$, YUJUN ZHAO ${ }^{2}, \mathrm{CHEN} \mathrm{LI}^{2}$ and XIN LI ${ }^{1}$ \\ ${ }^{1}$ Department of Neurology, The Second Hospital of Tianjin Medical University, Tianjin 300211; ${ }^{2}$ Department of Neurology; \\ ${ }^{3}$ Central Laboratory; ${ }^{4}$ Department of Pathology, The Fifth Central Hospital of Tianjin, Tianjin 300450, P.R. China
}

Received October 16, 2016; Accepted June 13, 2017

DOI: $10.3892 / \mathrm{mmr} .2017 .7425$

\begin{abstract}
Acute cerebral infarction can progress rapidly, and there are limited specific and effective treatments. Small ubiquitin-like modifiers (SUMOs) provide an important post-translational modification of proteins. Following cerebral infarction, multiple proteins can combine with SUMOs to protect nerve cells. Furthermore, moderate hypothermia (core body temperature, $33-34^{\circ} \mathrm{C}$ ) can increase the level of SUMOylation on multiple proteins. In the present study, it was examined whether moderate hypothermia increases the survival rate of bone marrow stromal stem cells (BMSCs) implanted in the cerebral ischemic penumbra via SUMOylation of multiple proteins. Firstly, BMSCs were exposed to oxygen-glucose deprivation (OGD) under moderate hypothermic $\left(33^{\circ} \mathrm{C}\right)$ conditions. Subsequently, adult rats with middle cerebral artery occlusion were treated with a combination of BMSCs and moderate hypothermia $\left(32-34^{\circ} \mathrm{C}\right)$. The results demonstrated that hypothermia promoted the combination of multiple proteins with SUMOs in BMSCs, and induced transport of SUMOs from the cytoplasm to the nucleus. Moderate hypothermia additionally reduced damage to BMSCs following OGD and improved BMSC survival following transplantation into the penumbra. These data suggest that moderate hypothermia may protect against BMSC injury via rapid SUMOylation of intracellular proteins. Thus, BMSC transplantation combined with moderate hypothermia may be a potential therapeutic strategy to treat cerebral infarction.
\end{abstract}

Correspondence to: Mrs. Xin Li, Department of Neurology, The Second Hospital of Tianjin Medical University, 23 Pingjiang Road, Hexi, Tianjin 300211, P.R. China

E-mail: jessielx@126.com

Key words: small ubiquitin-like modifiers, SUMOylation, moderate hypothermia, bone marrow stromal stem cells, infarction, cerebral ischemic penumbra

\section{Introduction}

Cerebrovascular ischemia is challenging to treat due to its rapid progression, and there are a limited number of effective treatments. The resulting cerebral infarction can cause severe sequelae in patients and markedly affects quality of life (1). The penumbral areas around the main cerebral lesions contain numerous dormant or semi-dormant brain cells that can only maintain their integrity, rather than perform their normal function, as a result of reduced energy and oxygen supply (2). The protection of these cells is important for clinical treatment of infarction (3). Bone marrow stromal cells (BMSCs) are skeletal progenitor cells that can differentiate into bone, cartilage, fat and nerve cells $(4,5)$. BMSCs are easy to acquire, expand, genetically manipulate and transplant in vivo, and are becoming increasingly used in the field of neural regeneration and transplantation (6).

Moderate hypothermia is well recognized to protect the brain from hypoxic-ischemic injury, potentially via reducing the cerebral metabolic rate of oxygen and lowering the synthesis and release of excitotoxic neurotransmitters and inflammatory mediators $(7,8)$. The penumbral region of the brain is an ischemic, hypoxic, inflammatory and toxic environment, which limits stem cell transplantation into the penumbra due to their reduced survival and proliferation, in addition to premature aging (9-11). The combination of neural stem cell transplantation into the penumbra and moderate hypothermia may provide an improved treatment regime for ischemic stroke (12). However, the molecular mechanisms of protection by the combination neural stem cells and hypothermia remain unclear. Understanding these mechanisms is important to provide more effective and rational clinical treatments involving moderate hypothermia.

Small ubiquitin-related modifiers (SUMOs) are an important class of post-translational modification protein factors and involved in maintaining genome stability (13), protein-protein interactions, translocation between the cytoplasm and nucleus, and limiting ubiquitination by combining with target proteins $(14,15)$. SUMO modification is a dynamic and reversible event in cells, suggesting that certain SUMOylation-associated pathological events may be reversible. Notably, cerebral ischemia has been reported to induce SUMOylation in neuronal cells with increased binding of 
SUMOs to target proteins, thereby performing a protective role $(16,17)$. Thus, it was hypothesized that the neuroprotective actions of moderate hypothermia following cerebral infarction may be associated with increased SUMOylation of multiple proteins in neurons.

\section{Materials and methods}

Experimental animals. A total of 40 12-week-old (307士28.3 g) and 2 newborn (5.1 $\pm 0.62 \mathrm{~g})$ Sprague-Dawley rats were purchased from the Animal Center of the Cancer Institute of the Chinese Academy of Medical Science (Beijing, China). Animals were housed in the Animal Experimental Center of the Fifth Central Hospital of Tianjin (Tianjin, China) at $20-25^{\circ} \mathrm{C}$ with $50 \pm 5 \%$ humidity. All experiments were performed according to the Principles of Laboratory Animal Care (18) and was approved by the Ethics Committee of The Fifth Central Hospital of Tianjin.

Cell culture. Methods for the extraction and culture of BMSCs have been described previously $(4,19)$. Briefly, the long bones of the hind legs were dissected from the two newborn male rats, and bone marrow plugs were extracted from the bones by flushing the bone marrow cavity with complete culture medium (RASMX-90011, Cyagen Biosciences Inc. Santa Clara, CA, USA). About $5 \times 10^{7}$ cells were seeded in a $75-\mathrm{cm}^{2}$ culture flask and incubated at $37^{\circ} \mathrm{C}$ in a humidified atmosphere with $5 \% \mathrm{CO}_{2}$. Non-adherent cells were removed after $24 \mathrm{~h}$, and the culture medium was replaced every 3 days. Adherent cells reached $90-95 \%$ confluence within 10-15 days and were passaged with $0.25 \%$ trypsin (Invitrogen; Thermo Fisher Scientific, Inc., Waltham, MA, USA) at a ratio of 1:3. Cells at passage 3 were characterized by flow cytometry (Abcam, Shanghai, China).

Oxygen-glucose deprivation model and moderate hypothermic therapy. To simulate cerebral ischemia, BMSCs were cultured in an anoxic chamber (Forma Scientific Anaerobic System; Thermo Fisher Scientific, Inc.) (20). Glucose-, L-aspartic acid-, L-glutamic acid-, and sodium pyruvate-free neurobasal medium (Gibco; Thermo Fisher Scientific, Inc.) was equilibrated overnight in the anoxic chamber with the anoxic gas mixture $\left(85 \% \mathrm{~N}_{2}, 10 \% \mathrm{H}_{2}\right.$ and $\left.5 \% \mathrm{CO}_{2}\right)$. Cultured cells were washed three times with the anoxic medium and then transferred to the anoxic chamber. Following $60 \mathrm{~min}$ of oxygen-glucose deprivation (OGD), the anoxic medium was replaced with neurobasal/B27 medium, and the cells were transferred to incubators set at $33^{\circ} \mathrm{C}$ or $37^{\circ} \mathrm{C}$ with a gas mixture of $95 \%$ air and $5 \% \mathrm{CO}_{2}$ for an additional $24 \mathrm{~h}$. The neurobasal/B27 medium was pre-warmed to $33^{\circ} \mathrm{C}$ or $37^{\circ} \mathrm{C}$ for induction of moderate hypothermia.

Small interfering RNA transfection. A small interfering RNA (siRNA) duplex targeted to the Ubc9 gene was purchased from Shanghai GenePharma Co., Ltd. (Shanghai, China) (sense, 5'GGGAAGGACCCTTGTTTAA3'; anti-sense, 5'CTT AAAGGCGTTCGTTAGG3'). Lipofectamine RNAiMAX transfection reagent (Thermo Fisher Scientific, Inc.) was used according to the manufacturer's instructions. SiUBC9 and a universal negative control (SiNC) were purchased from
Shanghai GenePharma Co., Ltd. SiUBC9 and SiNC were applied at $10 \mathrm{nM}$ dissolved in Opti-MEM (Thermo Fisher Scientific, Inc.).

Immunofluorescence. BMSCs were seeded onto glass slides and cultured at $33^{\circ} \mathrm{C}$ for 2 or $24 \mathrm{~h}$. The cells were then fixed in $4 \%$ paraformaldehyde/phosphate-buffered saline (PBS), blocked with 20\% goat serum (Gibco; Thermo Fisher Scientific, Inc.), and incubated with anti-SUMO1 (ab11672; 1:1,000; Abcam, Cambridge, MA, USA) and anti-SUMO2/3 (ab3742; 1:500; Abcam) antibodies at $4^{\circ} \mathrm{C}$ overnight. Then, the cells were incubated in Fluorescein (FITC) or Texas Red-labeled secondary antibodies (sc-2012 or sc-3917; 1:500; Santa Cruz Biotechnology, Santa Cruz, CA, USA), with PBST for $3 \mathrm{~min}$ and washed 3 times; followed by 4,6-diamidino-2-phenylindole to stain cell nuclei for $5 \mathrm{~min}$, darkening the specimen, and washed 4 times with PBST. Image Pro Plus 6.0 software (Media Cybernetics, Rockville, MD, USA) was used for semi quantitative analysis. The light densities of the nucleus and cytoplasm were measured. A result was considered as positive when the ratio was $>1$, and then the positive rate was calculated.

Western blot analysis. To avoid de-SUMOylation of proteins during sample preparation, BMSCs were homogenized in ice-cold RIPA lysis buffer (EMD Millipore, Billerica, MA, USA). The cell lysates were centrifuged at 17,970 x $\mathrm{g}$ for $15 \mathrm{~min}$ at $4^{\circ} \mathrm{C}$. The supernatant was collected, and protein concentrations were measured using a bicinchoninic acid assay kit (Thermo Fisher Scientific, Inc.). Samples were mixed with sample buffer (Invitrogen; Thermo Fisher Scientific, Inc.), incubated at $70^{\circ} \mathrm{C}$ for $10 \mathrm{~min}$, and then applied to sodium dodecyl sulfate polyacrylamide gel electrophoresis (4-15\% gels; Invitrogen; Thermo Fisher Scientific, Inc.) with $30 \mu \mathrm{g}$ total protein added per well. Subsequent to electrophoresis, the proteins were transferred to polyvinylidene fluoride membranes (EMD Millipore). The membranes were blocked in a Tris-HCl-buffered salt solution containing $0.1 \%$ Tween-20 (TBST) and 5\% skimmed milk powder, and then incubated with anti-SUMO1 $(1: 1,000)$, anti-SUMO2/3 $(1: 1,000)$, or anti-UBC9 (cat. no. 4786; 1:1,000; Cell Signaling Technology, Inc., Danvers, MA, USA) antibodies overnight at $4^{\circ} \mathrm{C}$. Then, the membranes were washed five times with TBST, followed by incubation with horseradish peroxidase-conjugated goat anti-rabbit IgG (111-035-003; 1:2,000; Jackson Immuno Research Laboratories, Inc., West Grove, PA, USA) as the secondary antibody for $1 \mathrm{~h}$ at room temperature. Following five washes with $0.1 \%$ TBST, proteins were detected using a C-Digit Blot Scanner (LI-COR Biosciences, Lincoln, NE, USA). The membrane was then stripped and re-incubated with an anti- $\beta$-actin primary antibody (cat. no. 3700; 1:1,000; Cell Signaling Technology, Inc.). Data were evaluated by image analysis software (ImageJ version 1.48; National Institutes of Health, Bethesda, MD, USA).

Apoptosis detection. BMSCs were harvested, washed with PBS, immersed in permeabilization solution for $5 \mathrm{~min}$, and then incubated with $25 \mu \mathrm{l}$ terminal deoxynucleotidyl transferase dUTP nick end labeling (TUNEL) reaction mixture (Sigma-Aldrich; Merck Millipore, Darmstadt, Germany) in a 


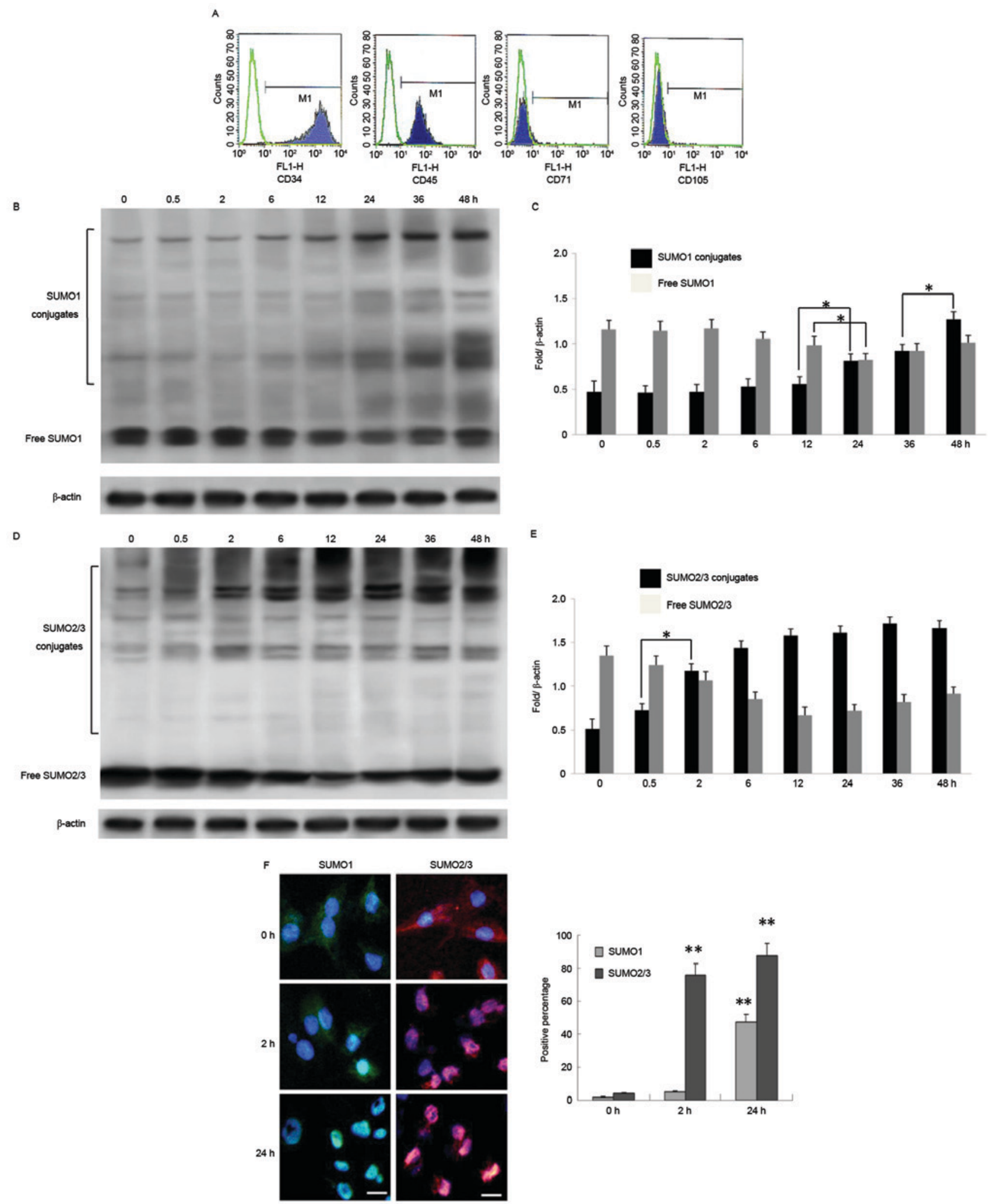

Figure 1. Moderate hypothermia promotes expression of SUMO1 and SUMO2/3 in BMSCs and induces their nuclear translocation. (A) BMSC-specific proteins were detected by flow cytometry. (B and C) Time course of SUMO1 and SUMO2/3 conjugate and free SUMO1 and SUMO2/3 expression in BMSCs after moderate hypothermic treatment, as assessed by western blotting. $\beta$-actin was used for normalization $(n=3)$. ( $D$ and $E$ ) Ratios of target proteins to $\beta$-actin $\left(\mathrm{n}=3\right.$ ). ${ }^{*} \mathrm{P}<0.05$. (F) Photomicrographs of SUMO1 (green), SUMO2/3 (red), and nuclei (blue) in BMSCs treated at $33^{\circ} \mathrm{C}$ for 0,2 and $24 \mathrm{~h}$. Scale bars, $10 \mu \mathrm{m}$. ${ }^{* *} \mathrm{P}<0.01$, BMSCs treated at $33^{\circ} \mathrm{C}$ for 2 and $24 \mathrm{~h}$ vs. $0 \mathrm{~h}$ within one group. Data are expressed as the mean \pm standard error. SUMO, small ubiquitin-related modifier; BMSCs, bone marrow stromal cells.

humidified chamber at $37^{\circ} \mathrm{C}$ for $60 \mathrm{~min}$. Subsequent to washing with PBS, nuclei were counterstained with Hoechst 33258
(Sigma-Aldrich; Merck Millipore). The samples were then washed with PBS and deionized water, mounted and observed 
under a fluorescence microscope (Olympus Corporation, Tokyo, Japan). The total number of cells (Hoechst-positive) and the number of apoptotic cells (TUNEL-positive) were quantified. The apoptotic rate (\%) was calculated by the number of apoptotic cells/the number of total cells x $100 \%$.

Lactate dehydrogenase $(\mathrm{LDH})$ activity detection. After harvesting the BMSCs, LDH content in the culture media was measured by an enzyme-linked immunosorbent assay (LDH Activity Assay kit; BioVision, Inc., San Francisco, CA, USA) according to the manufacturer's protocols.

Rat middle cerebral artery occlusion model and moderate hypothermic treatment. A total of 40 adult male Sprague-Dawley rats were randomly divided into four groups. For surgery, animals were maintained by a small animal ventilator (Shanghai Yuyan Instruments Co., Ltd., Shanghai, China), and rectal temperature was monitored to control body temperature. A $1 \mathrm{~cm}$ longitudinal incision was made between the sternum and mandible, the left common carotid artery was isolated, external carotid and internal carotid arteries were visualized under a microscope (Olympus Corporation), the proximal heart end of the carotid artery and distal heart end of the external carotid artery were ligated, and a nylon suture (head-end diameter, $0.23 \mathrm{~mm}$; trunk diameter, $0.18 \mathrm{~mm}$ ) was inserted from the carotid artery into the middle cerebral artery ( $12.0 \mathrm{~mm}$ deep) and fixed with a suture.

BMSCs or SiUBC9 BMSCs were transplanted into the ischemic penumbra after establishment of the middle cerebral artery occlusion (MCAO) models as reported previously $(12,21,22)$. To induce moderate hypothermia, rats transplanted with BMSCs were placed on a hypothermia blanket (Shanghai Yuyan Instruments Co., Ltd.), and a rectal temperature monitor was used to control body temperature at $32-34^{\circ} \mathrm{C}$ for $12 \mathrm{~h}$. The animals were then removed from anesthesia and gradually returned to normal body temperature. Animals were recovered for 2, 7, 14 or 21 days, and neurological functions were assessed by neurological severity scores (NSSs) $(23,24)$.

Statistics analysis. All experiments were repeated at least three times. Data are expressed as the mean \pm standard error. Statistical software (GraphPad Prism 6; GraphPad Software, Inc., La Jolla, CA, USA) was used for all statistical tests. Comparisons between multiple groups were evaluated by one-way analysis of variance, and comparisons between two groups were evaluated by Student's unpaired t-test. $\mathrm{P}<0.05$ was considered to indicate a statistically significant difference.

\section{Results}

Moderate hypothermia promotes SUMO1 and SUMO2/3 binding to target proteins and induces SUMO translocation from the cytoplasm to the nucleus in BMSCs. BMSCs extracted from rats were cultured in conditioned medium and identified by flow cytometry. The results indicated high levels of expression of BMSC-specific proteins CD71 and CD105, however no expression of hematopoietic stem cell-specific proteins CD34 and CD45 (Fig. 1A). BMSCs were then cultured at $33^{\circ} \mathrm{C}$ for $0-48 \mathrm{~h}$ and used to detect expression of
SUMO1 and SUMO2/3. There was a trend towards a slow increase in expression of free SUMO1 conjugates up to $24 \mathrm{~h}$ after moderate hypothermia $\left(33^{\circ} \mathrm{C}\right)$, a significant decrease after $24 \mathrm{~h}$, and a marginal increase at $48 \mathrm{~h}$. It was suggested that, following $36-48 \mathrm{~h}$ of moderate hypothermia, production of new free SUMO1 may exert cytoprotective effects (Fig. 1B and C). In contrast, short term treatment of BMSCs with moderate hypothermia $(<2 \mathrm{~h})$ induced a trend towards an increase in combined SUMO2/3 expression, which peaked at 24-36 h, and then remained elevated after $36 \mathrm{~h}$ (Fig. 1D and E). BMSCs cultured under moderate hypothermia for 2 or $24 \mathrm{~h}$ also presented with marked translocation of SUMO1 and SUMO2/3 from the cytoplasm to the nucleus (SUMO1 at $24 \mathrm{~h}$ and SUMO2/3 at $2 \mathrm{~h}$; Fig. $1 \mathrm{E}$ and F).

SiUBC9 inhibits SUMO binding to target proteins in BMSCs under hypoxia. UBC9 is the SUMO-conjugating enzyme E2 that acts on inactive SUMOs to induce SUMOylation of substrates together with the SUMO-activating enzyme E1, SUMO ligase E3, and adenosine triphosphate (25). In the present study, BMSCs were cultured under OGD to simulate hypoxia-ischemia. Using RNA interference in BMSCs, expression of UBC9 was significantly reduced compared with wild-type (WT) and SiNC BMSCs (Fig. 2A and B). Following OGD, expression of SUMO conjugates and free SUMOs was significantly stimulated in WT and SiNC BMSCs. Conversely, in UBC9 knockdown BMSCs, SUMO1 and SUMO2/3 conjugates were reduced significantly, while the level of free SUMOs was significantly increased under normal and OGD culture conditions (Fig. 2C-F).

Moderate hypothermia causes SUMOylation-dependent reductions in BMSC apoptosis and $\mathrm{LDH}$ secretion following hypoxia. WT and SiNC BMSCs cultured at $33^{\circ} \mathrm{C}$ following OGD exhibited significant decreases in apoptosis and LDH secretion compared with those cultured at $37^{\circ} \mathrm{C}$. However, UBC9 knockdown BMSCs exhibited no resistance against moderate hypothermia (Fig. 3A and B). Thus, a lack of UBC9 hinders SUMO binding to target proteins, it is speculated that the protective effect of moderate hypothermia may be associated with intracellular protein SUMOylation. Notably, down-regulation of UBC9 expression was additionally observed in BMSCs under normal culture conditions, which was associated with an increase in BMSC apoptosis and $\mathrm{LDH}$ release, suggesting a role of protein SUMOylation in the maintenance of cellular functions and resistance. These results indicated that knockdown of UMBC9 reduced the binding ability of SUMOs to target proteins and suppressed the cytoprotective effect.

Moderate hypothermia causes SUMOylation-dependent improvements in survival of BMSCs transplanted into the ischemic penumbra. WT and SiUBC9 BMSCs were transplanted into the penumbra of MCAO model rats that were then subjected to moderate hypothermia. There was a progressive reduction in neurological function as assessed by the NSSs of MCAO animals without BMSC transplantation or moderate hypothermia. In contrast, MCAO rats with BMSC transplantation had improved neurological functions, whereas animals with BMSC transplantation combined with moderate 
Table I. Comparison of neurological severity scores at different times between groups.

\begin{tabular}{lccccc}
\hline Neurological severity scores & $\mathrm{n}$ & \multicolumn{1}{c}{2 days } & \multicolumn{1}{c}{7 days } & 14 days & 21 days \\
\hline MCAO & 10 & $34.14 \pm 3.47$ & $28.19 \pm 4.02$ & $20.41 \pm 3.78$ & $17.43 \pm 3.55$ \\
$37^{\circ} \mathrm{C}$ BMSCs & 10 & $28.49 \pm 3.45^{\mathrm{a}}$ & $20.58 \pm 3.09^{\mathrm{a}}$ & $15.45 \pm 2.38^{\mathrm{a}}$ & $14.67 \pm 2.11^{\mathrm{a}}$ \\
$33^{\circ} \mathrm{C}$ BMSCs & 10 & $27.15 \pm 2.47^{\mathrm{a}, \mathrm{b}}$ & $17.49 \pm 2.19^{\mathrm{a}, \mathrm{b}}$ & $12.58 \pm 2.16^{\mathrm{a}, \mathrm{b}}$ & $10.43 \pm 1.93^{\mathrm{a}}$ \\
$33^{\circ} \mathrm{C}$ BMSCs SiUBC9 & 10 & $28.11 \pm 2.72^{\mathrm{b}}$ & $19.14 \pm 3.01^{\mathrm{b}}$ & $14.09 \pm 2.33$ & $12.45 \pm 2.96$
\end{tabular}

MCAO rats treated with BMSCs and moderate hypothermia. MCAO, MCAO model without treatment; $37^{\circ} \mathrm{C}$ BMSCs, $\mathrm{MCAO}$ models with WT BMSC transplantation; $33^{\circ} \mathrm{C}$ BMSCs, MCAO model with WT BMSC transplantation and $33^{\circ} \mathrm{C}$ hypothermic treatment for $24 \mathrm{~h}$; $33^{\circ} \mathrm{C}$ BMSCs SiUBC9, MCAO models with SiUNC9 BMSC transplantation and $33^{\circ} \mathrm{C}$ hypothermic treatment for $24 \mathrm{~h}$. Neurological scores were obtained at 2, 7,14 and 21 days following surgery in each group. Data are expressed as the mean \pm standard error $(n=10)$. ${ }^{a} \mathrm{P}<0.05$ vs. MCAO, ${ }^{\mathrm{b}} \mathrm{P}<0.05$ vs. $37^{\circ} \mathrm{C}$ BMSCs. MCAO, middle cerebral artery occlusion; BMSCs, bone marrow stromal cells; WT, wild-type; Si, small interfering.

A
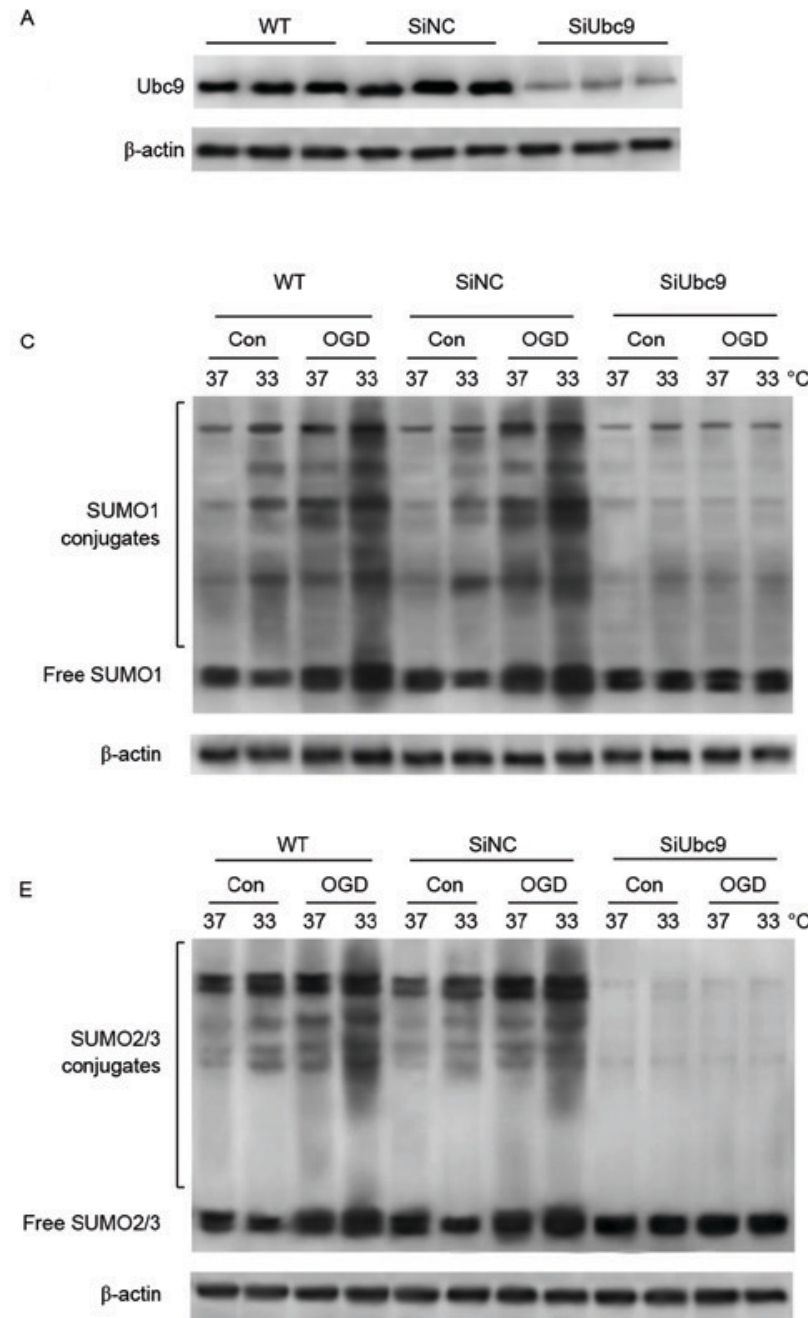

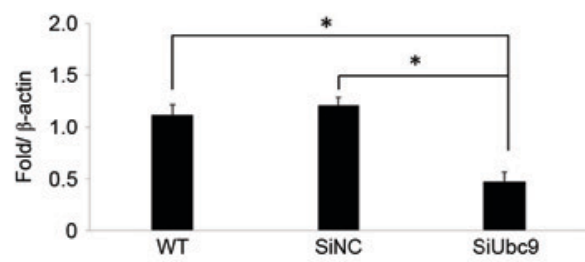

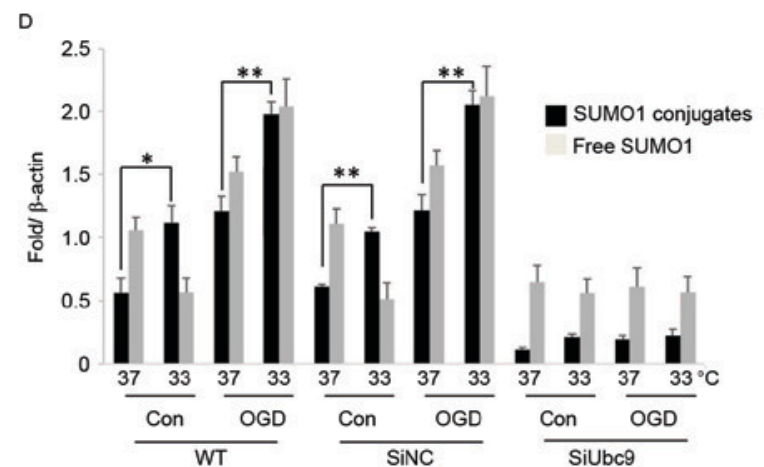

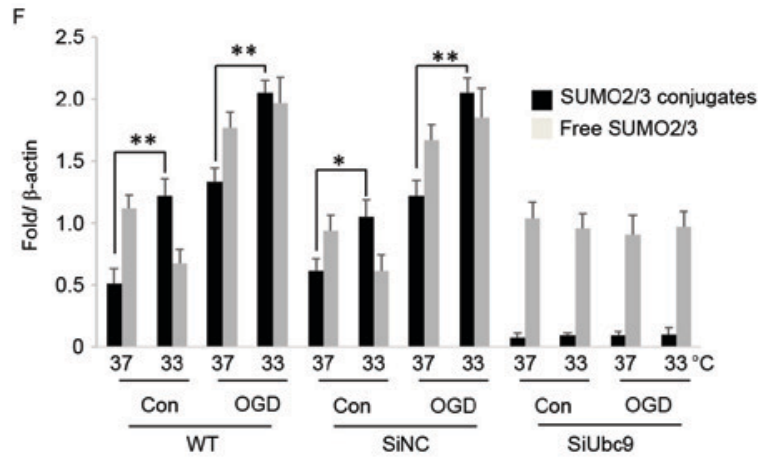

Figure 2. SiUBC9 blocks binding of SUMOs to target proteins following OGD in BMSCs. (A) UBC9 expression measured by western blotting. $\beta$-actin was used for normalization $(n=3)$. (B) Ratios of target proteins to $\beta$-actin $(n=3)$. Data are expressed as the mean \pm standard error; ${ }^{*} \mathrm{P}<0.05$. (C) Expression of SUMO1 conjugates and free SUMO1 in WT, SiNC, and SiUBC9 BMSCs following OGD. $\beta$-actin was used for normalization (n=3). (D) Ratios of SUMO1 conjugates and free SUMO1 to $\beta$-actin. Data are expressed as the mean \pm standard error $(n=3)$. (E) Expression of SUMO2/3 conjugates and free SUMO2/3 in WT, SiNC, and SiUBC9 BMSCs following OGD. $\beta$-actin was used for normalization $(n=3)$. (F) Ratios of SUMO2/3 conjugates and free SUMO2/3 to $\beta$-actin. Data are expressed as the mean \pm standard error $(n=3)$. ${ }^{*}<<0.05,{ }^{* *} \mathrm{P}<0.01$. Si, small interfering; SUMO, small ubiquitin-related modifier; BMSCs, bone marrow stromal cells; OGD, oxygen-glucose deprivation; WT, wild-type; NC, negative control; Con, control.

hypothermia showed further improvements. However, the improvement in neurological functions of MCAO rats that received transplantations of BMSCs with UBC9 knockdown following moderate hypothermia was less than that in animals transplanted with BMSCs at $33^{\circ} \mathrm{C}$ (Table I). These data suggest that transplantation of BMSCs into the penumbra 


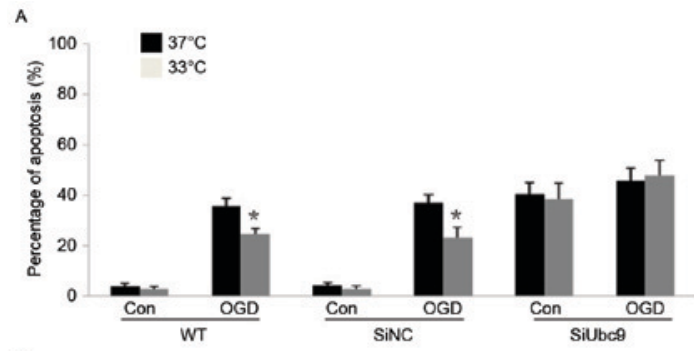

B

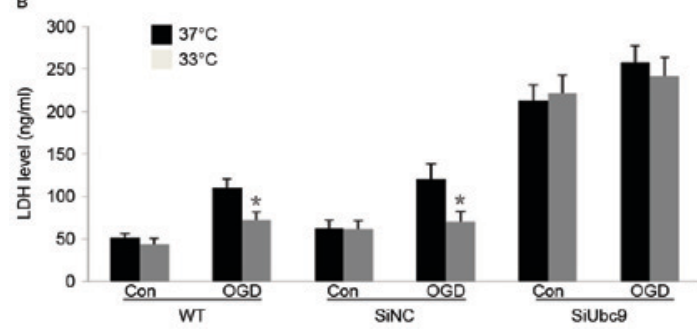

Figure 3. SiUBC9 inhibits the protective effect of moderate hypothermia on BMSCs after OGD. (A) WT, SiNC, and SiUBC9 BMSCs were cultured for $24 \mathrm{~h}$ after OGD treatment and then cultured at 33 or $37^{\circ} \mathrm{C}$ for $24 \mathrm{~h}$. The percentage of apoptotic BMSCs is presented. Data are expressed as the mean \pm standard error $(n=4) .{ }^{\prime \prime} \mathrm{P}<0.05$, treated at $33^{\circ} \mathrm{C}$ vs. $37^{\circ} \mathrm{C}$ within one group. (B) WT, SiNC and SiUBC9 BMSCs were cultured for $24 \mathrm{~h}$ after OGD and then cultured at 33 or $37^{\circ} \mathrm{C}$ for $24 \mathrm{~h}$. The $\mathrm{LDH}$ level in the culture medium is presented. Data are expressed as the mean \pm standard error $(n=4)$ ${ }^{*} \mathrm{P}<0.05$, treated at $33^{\circ} \mathrm{C}$ vs. $37^{\circ} \mathrm{C}$ within one group. $\mathrm{Si}$, small interfering, BMSCs, bone marrow stromal cells; OGD, oxygen-glucose deprivation; WT, wild-type; $\mathrm{NC}$, negative control; Con, control; $\mathrm{LDH}$, lactate dehydrogenase.

improves neurological function following MCAO, and that moderate hypothermia increases the activity and survival of transplanted BMSCs and promotes functional recovery, potentially via increased intracellular protein SUMOylation.

\section{Discussion}

There has been a progressive increase in the incidence of cerebral infarction due to the increasing rates of diabetes, hypertension, and high cholesterol in the general population. Early interventional therapy is an effective treatment for cerebral ischemia. However, the majority of patients are not treated within this time window, resulting in serious sequelae. Thus, new treatments are urgently required, which are effective outside of this time window. BMSCs are skeletal progenitor cells that are easy to obtain, expand in vitro, and transplant in vivo. Previous studies have demonstrated that BMSCs can be differentiated into bone, cartilage, fat and nerve cells, and that BMSCs may be neuroprotective (26-28). Thus, transplantation of BMSCs into the infarction area and subsequent differentiation into neuronal cells may provide a novel treatment for cerebral infarction. However, the hypoxic-ischemic environment in brain tissue following infarction can markedly reduce the survival of transplanted cells (29).

SUMOs are a class of important ubiquitin-like proteins. There are four SUMO family members that have been identified in humans (SUMO1-4), by contrast, the SUMO-conjugating enzyme E2 has only one form (UBC9), and RNA interference knockdown of UBC9 expression can effectively block SUMOylation of intracellular proteins (30). SUMOs have both free and conjugate forms that maintain the balance of cellular
SUMOylation. Protein SUMOylation serves an important role in maintenance of genomic stability, regulation of protein interactions, nuclear/cytoplasmic translocation, transcription factor activity, and inhibition of protein biotinylation. Notably, proteomic studies have identified that, following cerebrovascular ischemia, numerous proteins closely associated with physiological and pathological processes, including oxidative stress, inflammation, DNA synthesis, energy transfer and metabolism, are SUMOylated, which may aid in the removal of foreign material, reduce inflammatory responses, and regulate cellular proliferation and apoptosis $(31,32)$. In addition, it was observed that SUMO2/3 are neuroprotective and improve behavioral outcomes following experimental cerebral ischemia $(17,33)$.

Moderate hypothermic treatment is an effective therapy for treatment of high intracranial pressure and brain injury caused by severe hypoxia-ischemia. Hypothermia reduces the cellular metabolic rate, increases energy reserves to allow cellular recovery, reduces the inflammatory response and improves the brain microenvironment (34). In the present study, the association between moderate hypothermic treatment and protein SUMOylation was observed in BMSCs following hypoxia-ischemia. It was identified that conjugated SUMO2/3 were rapidly and highly induced in BMSCs subsequent to moderate hypothermic treatment, while free SUMO2/3 were decreased with marked SUMO2/3 nuclear translocation. These data suggest that the protective actions of moderate hypothermia are associated with the binding of SUMO2/3 to target proteins, rather than the initial expression of SUMO2/3 (35). In contrast, there was slow and low level SUMOylation of target proteins by SUMO1 with only moderate nuclear translocation. Further studies are required to determine the specific target proteins that combine with SUMOs and their biological functions in the SUMOylation pathway.

It was observed that the levels of conjugated SUMO1 and SUMO2/3, in addition to their nuclear translocation, were progressively increased in BMSCs following OGD treatment (SUMO2/3 within $2 \mathrm{~h}$ and SUMO1 from 24-48 h). Similar results have been reported previously (33). The rate of BMSC apoptosis and LDH secretion induced by OGD treatment were also reduced by moderate hypothermic treatment. Furthermore, these effects disappeared following UBC9 knockdown in BMSCs, which blocks the binding of SUMOs to their target proteins (36). Finally, the effects of BMSCs, moderate hypothermia and combination therapy on the recovery of neurological function in MCAO rats were investigated. Results indicated that transplantation of BMSCs into the ischemic penumbra in MCAO rats combined with moderate hypothermia was optimal for restoring neurological function. Furthermore, this protection was SUMOylation-dependent, explained by the fact that the improvement in neurological function in MCAO rats receiving transplantation of BMSCs with UBC9 knockdown following moderate hypothermia had a reduced quality of neurological function than animals transfected with BMSCs at the same temperature.

In summary, moderate hypothermia significantly enhances the ability of SUMOs to bind to their target proteins in BMSCs, suggesting an important role in regulating cellular apoptosis and functions following hypoxia-ischemia. Thus, moderate hypothermia promotes neural stem cell survival in the 
ischemic penumbra by increasing SUMOylation of multiple proteins. The results provide a further understanding of the molecular mechanisms underlying the protective actions of moderate hypothermia, and provide a theoretical basis for the use of bone marrow stem cell transplantation combined with moderate hypothermia for treatment of cerebral infarction.

\section{Acknowledgements}

The current study was supported by the National Natural Science Foundation of China (grant no. 81471175), Tianjin Health Bureau Science and Technology Projects (grant no. 2014KY23) and Tanggu Science and Technology Promotion Projects (grant no. 201 3KJXQ03).

\section{References}

1. van Rooy MJ and Pretorius E: Obesity, hypertension and hypercholesterolemia as risk factors for atherosclerosis leading to ischemic events. Curr Med Chem 21: 2121-2129, 2014.

2. An H, Ford AL, Chen Y, Zhu H, Ponisio R, Kumar G, Shanechi AM, Khoury N, Vo KD, Williams J, et al: Defining the ischemic penumbra using magnetic resonance oxygen metabolic index. Stroke 46: 982-988, 2015.

3. Kirton A and Deveber G: Life after perinatal stroke. Stroke 44: 3265-3271, 2013.

4. Baksh D, Song L and Tuan RS: Adult mesenchymal stem cells: Characterization, differentiation, and application in cell and gene therapy. J Cell Mol Med 8: 301-316, 2004.

5. Black IB and Woodbury D: Adult rat and human bone marrow stromal stem cells differentiate into neurons. Blood Cells Mol Dis 27: 632-636, 2001.

6. Chen JR, Cheng GY, Sheu CC, Tseng GF, Wang TJ and Huang YS: Transplanted bone marrow stromal cells migrate, differentiate and improve motor function in rats with experimentally induced cerebral stroke. J Anat 213: 249-258, 2008.

7. Campos F, Blanco M, Barral D, Agulla J, Ramos-Cabrer P and Castillo J: Influence of temperature on ischemic brain: Basic and clinical principles. Neurochemistry Int 60: 495-505, 2012.

8. Froehler MT and Ovbiagele B: Therapeutic hypothermia for acute ischemic stroke. Expert Rev Cardiovasc Ther 8: 593-603, 2010.

9. Eckert MA, Vu Q, Xie K, Yu J, Liao W, Cramer SC and Zhao W: Evidence for high translational potential of mesenchymal stromal cell therapy to improve recovery from ischemic stroke. J Cereb Blood Flow Metab 33: 1322-1334, 2013.

10. Yu SP, Wei Z and Wei L: Preconditioning strategy in stem cell transplantation therapy. Transl Stroke Res 4: 76-88, 2013.

11. Shinozuka K, Dailey T, Tajiri N, Ishikawa H, Kim DW, Pabon M, Acosta S, Kaneko Y and Borlongan CV: Stem Cells for Neurovascular Repair in Stroke. J Stem Cell Res Ther 4: 12912,2013

12. Tu Y, Chen C, Sun HT, Cheng SX, Liu XZ, Qu Y, Li XH and Zhang S: Combination of temperature-sensitive stem cells and mild hypothermia: A new potential therapy for severe traumatic brain injury. J Neurotrauma 29: 2393-2403, 2012.

13. Yurchenko V, Xue Z and Sadofsky MJ: SUMO modification of human XRCC4 regulates its localization and function in DNA double-strand break repair. Mol Cell Biol 26: 1786-1794, 2006.

14. Cubenas-Potts $C$ and Matunis MJ: SUMO: A multifaceted modifier of chromatin structure and function. Dev Cell 24: 1-12, 2013.

15. Bossis G and Melchior F: Regulation of SUMOylation by reversible oxidation of SUMO conjugating enzymes. Mol Cell 21: 349-357, 2006.

16. Silveirinha V, Stephens GJ and Cimarosti H: Molecular targets underlying SUMO-mediated neuroprotection in brain ischemia. J Neurochem 127: 580-591, 2013.

17. Yang W, Sheng H, Homi HM, Warner DS and Paschen W: Cerebral ischemia/stroke and small ubiquitin-like modifier (SUMO) conjugation-a new target for therapeutic intervention? J Neurochem 106: 989-999, 2008.
18. Trappe K, Thomas D, Bikou O, Kelemen K, Lugenbiel P, Voss F, Becker R, Katus HA and Bauer A: Suppression of persistent atrial fibrillation by genetic knockdown of caspase 3: A pre-clinical pilot study. Eur Heart J 34: 147-157, 2013.

19. Deng J, Petersen BE, Steindler DA, Jorgensen ML and Laywell ED: Mesenchymal stem cells spontaneously express neural proteins in culture and are neurogenic after transplantation. Stem Cells 24: 1054-1064, 2006.

20. Yang W, Thompson JW, Wang Z, Wang L, Sheng H, Foster MW, Moseley MA and Paschen W: Analysis of oxygen/glucose-deprivation-induced changes in SUMO3 conjugation using SILAC-based quantitative proteomics. J Proteome Res 11: 1108-1117, 2012.

21. Bleilevens C, Roehl AB, Goetzenich A, Zoremba N, Kipp M, Dang J, Tolba R, Rossaint R and Hein M: Effect of anesthesia and cerebral blood flow on neuronal injury in a rat middle cerebral artery occlusion (MCAO) model. Exp Brain Res 224: 155-164, 2013.

22. Cui L, Zhang X, Yang R, Liu L, Wang L, Li M and Du W: Baicalein is neuroprotective in rat MCAO model: Role of 12/15-lipoxygenase, mitogen-activated protein kinase and cytosolic phospholipase A2. Pharmacol Biochem Behav 96: 469-475, 2010

23. Zhang Y, Yang Y, Zhang GZ, Gao M, Ge GZ, Wang QQ, Ji XC, Sun YL, Zhang HT and Xu RX: Stereotactic Administration of Edaravone ameliorates collagenase-induced intracerebral hemorrhage in rat. CNS Neurosci Ther 22: 824-835, 2016.

24. Titova EM, Ghosh N, Valadez ZG, Zhang JH, Bellinger DL and Obenaus A: The late phase of post-stroke neurorepair in aged rats is reflected by MRI-based measures. Neuroscience 283: 231-244, 2014.

25. Johnson ES: Protein modification by SUMO. Annu Rev Biochem 73: 355-382, 2004.

26. Sindberg GM, Lindborg BA, Wang Q, Clarkson C, Graham M, Donahue R, Hering BJ, Verfaillie CM, Bansal-Pakala P and O'Brien TD: Comparisons of phenotype and immunomodulatory capacity among rhesus bone-marrow-derived mesenchymal stem/stromal cells, multipotent adult progenitor cells, and dermal fibroblasts. J Med Primatol 43: 231-241, 2014.

27. Maltman DJ, Hardy SA and Przyborski SA: Role of mesenchymal stem cells in neurogenesis and nervous system repair. Neurochem Int 59: 347-356, 2011.

28. Kurwale NS, Suri V, Srivastava A, Suri A, Mohanti S, Yadav P, Sharma MC and Sarkar C: Role of bone marrow derived pluripotent stem cells in peripheral nerve repair in adult rats: A morphometric evaluation. J Neurosci Rural Pract 6: 152-159, 2015.

29. Shinozuka K, Dailey T, Tajiri N, Ishikawa H, Kaneko Y and Borlongan CV: Stem cell transplantation for neuroprotection in stroke. Brain Sci 3: 239-261, 2013.

30. Sommer S, Ritterhoff T, Melchior F and Mootz HD: A stable chemical SUMO1-Ubc9 conjugate specifically binds as a thioester mimic to the RanBP2-E3 ligase complex. Chembiochem 16: 1183-1189, 2015.

31. Keusekotten $\mathrm{K}$ and Praefcke GJ: Reconstitution of SUMO-dependent ubiquitylation in vitro. Methods Mol Biol 832: 111-123, 2012.

32. Yang W, WangL and Paschen W: Development of ahigh-throughput screening assay for inhibitors of small ubiquitin-like modifier proteases. J Biomol Screen 18: 621-628, 2013.

33. Iwabuchi M, Sheng H, Thompson JW, Wang L, Dubois LG, Gooden D, Moseley M,Paschen W and Yang W: Characterization of the ubiquitin-modified proteome regulated by transient forebrain ischemia. J Cereb Blood Flow Metab 34: 425-432, 2014.

34. Sahuquillo J and Vilalta A: Cooling the injured brain: How does moderate hypothermia influence the pathophysiology of traumatic brain injury. Curr Pharm Des 13: 2310-2322, 2007.

35. Wang L, Ma Q, Yang W, Mackensen GB and Paschen W: Moderate hypothermia induces marked increase in levels and nuclear accumulation of SUMO2/3-conjugated proteins in neurons. J Neurochem 123: 349-359, 2012.

36. Lee YJ, Mou Y, Maric D, Klimanis D, Auh S and Hallenbeck JM: Elevated global SUMOylation in Ubc9 transgenic mice protects their brains against focal cerebral ischemic damage. PLoS One 6: e25852, 2011. 